\title{
Effect of dispersant viscosity for self-assembly process of barium titanate aggregates in material texture of polyvinylidene fluoride composites -evaluation of multifractal dimension $\left(D_{q}[q=0,1,2]\right)$ and dielectric properties-
}

\author{
Mariko TAKEDA $^{1, \dagger}{ }^{\dagger}$, Haruna YAMAZAKI ${ }^{1}$, Yoshihiro SATO $^{1}$, Makoto TANIMURA ${ }^{2}$, \\ Yasuhide INOUE $^{3}$, Yasumasa KOYAMA ${ }^{3}$ and Fumio MUNAKATA ${ }^{1}$ \\ ${ }^{1}$ Graduate School of Integrative Science and Engineering, Tokyo City University, \\ 1-28-1 Tamatsutsumi Setagaya-ku, Tokyo 158-8557, Japan \\ ${ }^{2}$ Research Initiatives and Promotion Organization, Instrumental Analysis Center, Yokohama National University, \\ 79-5 Tokiwadai, Hodogaya-ku, Yokohama 240-8501, Japan \\ ${ }^{3}$ Kagami Memorial Research Institute for Materials Science and Technology, Waseda University, \\ 2-8-26 Nishiwaseda, Shinjuku-ku, Tokyo 169-0051, Japan
}

In this paper, the properties of the barium titanate (BT)/polyvinylidene fluoride (PVDF) composites were controlled by the self-assembly of the BT secondary particle groups. The self-assembled BT/PVDF composites of 5-20 vol.\% BT were prepared with the difference in the viscosity of polyethylene glycol (PEG) of dispersants involved in the self-assembly process. The multifractal properties and dielectric properties of the self-assembled BT/PVDF composites were investigated with controlling by the formation of the self-assembled BT secondary particle groups. The dielectric constant $\left(\varepsilon^{\prime}\right)$ increased with an increase in the average secondary particle area $(S)$ of the BT particles. The $S$ increased with an increase in the viscosity of PEG; whereas, the $\varepsilon^{\prime}$ decreased with the increase in viscosity. The multifractal analysis indicated that the distribution of self-assembled BT secondary particle groups was affected by PEG viscosity. The sample with PEG1000 had aggregates with a BT/PVDF/BT heterointerface, the sample with PEG20000 had agglomerates with a BT/BT interface, and the sample with PEG2000 had both, aggregates and agglomerates. The BT/PVDF/BT heterointerface in the BT aggregates played an important role in improving the dielectric properties of the BT/PVDF composites. Overall, the viscosity of the dispersant affected the self-assembly process as well as the $\varepsilon^{\prime}$. It was suggested that the multifractal properties and dielectric properties were controlled by the self-assembly of the BT secondary particle groups.

(02022 The Ceramic Society of Japan. All rights reserved.

Key-words : $\mathrm{BaTiO}_{3}$, Self-assembly, Ceramics/Polymer composite, Multifractal, Dielectric properties, Dispersant

\section{Introduction}

Generally, the dielectric constant $\left(\varepsilon^{\prime}\right)$ of a polymer is relatively low at less than $10 .{ }^{1)}$ To increase the $\varepsilon^{\prime}$ for the energy storage in polymers, ceramic powder fillers with a high $\varepsilon^{\prime}$, such as lead oxide-based ferroelectrics and barium titanate $\left(\mathrm{BaTiO}_{3}, \mathrm{BT}\right)$ with a $\varepsilon^{\prime}$ of 1,000 or more, are added to the polymer matrix. ${ }^{1), 2)}$ The lead oxide-based ferroelectrics have excellent ferroelectric and piezoelectric

Corresponding author: M. Takeda; E-mail: marikotakeda1 @ gmail.com

$\$$ Preface for this article: Dol http://doi.org/10.2109/jcersj2. 130.P1-1 properties. However, lead oxide is the toxicity. ${ }^{2)}$ On the other hand, BT is one of the main lead-free electronics for dielectric, piezoelectric, and electro-optic applications. ${ }^{2)}$ Meanwhile, an example of using a polymer material with dielectric properties was provided in a previous report, ${ }^{3)}$ in which a polymer piezoelectric biosensor with a polyvinylidene fluoride (PVDF) was proposed. In this report, we proposed that it is possible to detect biopolymers using the adsorption characteristics of the host-guest reaction on the sensor via the relaxation behavior of the $\beta$-PVDF piezoelectric material. ${ }^{3)}$ However, $\beta$-PVDF has a significantly lower elastic constant than quartz, which is a signal transducer sensor of a general biosensor for mass detection. Therefore, the shear vibration of $\beta$-PVDF was 
affected by the solution viscosity, which, in turn, affected the change in the $\varepsilon^{\prime}$ of $\beta$-PVDF. ${ }^{3)}$ Hence, it is expected that it is possible to control the shear vibration by adding ceramic fillers to PVDF. In this paper, the BT/PVDF composites materials were manufactured as an application of BT. In general, a $50 \mathrm{vol} . \%$ or more of ceramics with a high $\varepsilon^{\prime}$ are applied to the polymer matrix to achieve a high $\varepsilon^{\prime}$. Thus, the mechanical properties of these composites are extremely poor and are not suitable for use in dielectric materials, such as capacitors, or piezoelectric materials.

Robertson and Varlow ${ }^{4}$ demonstrated that the size, shape, and aggregation of BT particles influence the effective bulk $\varepsilon^{\prime}$ of BT fillers or polymer composites with a high $\varepsilon^{\prime}$. Here the authors suggested the formation of agglomerations, which reduces the impedance due to the lower ratio of resin to filler across the shortest impedance path. ${ }^{4}$ Elsewhere, researchers have proposed a more considerable electrical flux for the movement of the agglomerated rather than the non-agglomerated particles, which will result in a higher permittivity for the per unit sample. ${ }^{4)}$ Meanwhile, Akimune et al. ${ }^{5)}$ conducted an analysis of the mechanical and electrical properties of $\mathrm{TiN} / \mathrm{Si}_{3} \mathrm{~N}_{4}$, in which the particle shape and size of the granules in the related ceramics play an important role in the network formation. Here, the authors highlighted that the formation of agglomerates improves functioning. ${ }^{5)}$ Phan et al. ${ }^{6}$ reported the effect of controlled BT nanoparticle aggregates on the dielectric properties of epoxy nanocomposites in terms of various experimental parameters, including frequency, ceramic content, and temperature. Here, the authors suggested that the nature of an interphase region depends not only on the chemical bonding at the filler interface but also on the network confinement between the nanoparticles. ${ }^{6)}$ More recently, researchers have also demonstrated that the average secondary particle area $(S)$ of the BT filler aggregates of BT filler/poly-L-lactic acid (PLLA) composites is related to the $\varepsilon^{\prime}$ in terms of regulating the microstructure of the aggregates. ${ }^{7)}$ In a previous report, we outlined how the BT/ PLLA/BT heterointerface formed within the kneading process via the self-assembly process and demonstrated that this has an important role in improving the $\varepsilon^{\prime}{ }^{8}$ )

A method incorporating fractal analysis was devised to quantitatively analyze the shape of particles, ${ }^{9)}$ with Kobayashi et al. ${ }^{10)}$ investigating the grain boundary microstructures in SUS316L stainless steel using the fractal analysis method. Here, the authors highlighted how the maximum random boundary connectivity was found to have a fractal nature in the SUS316L specimens and suggested that it was possible to characterize the grain boundary microstructures via fractal analysis. ${ }^{10)}$ In another of our previous reports, we investigated the characteristics of the aggregated morphology of the secondary particle group in BT/PLLA polymers using multifractal analysis. ${ }^{11)}$ Here we report that the self-assembled BT aggregate morphology had fractal characteristics and that the self-assembled BT aggregate morphology affected the dielectric properties. ${ }^{11)}$ In fact, recently, multifractal analysis, which is seen as an extension of fractal analysis, has been widely used in the medical field. ${ }^{12)}$ Here, Pérez et al. ${ }^{13)}$ reported the dispersion of $\mathrm{CaCO}_{3}$ particles in polypropylene (PP) and the fracture morphology of tensile samples using the boxcounting method and multifractal analysis (multifractal spectrum $(\alpha, f(\alpha))$. Here, the authors suggested that multifractal theory can be applied to elucidate the relationship between the structure and the mechanical behavior of PP$\mathrm{CaCO}_{3}$ composite materials. ${ }^{13)}$ Meanwhile, we previously estimated the multifractal dimensions of the $\beta-\mathrm{Si}_{3} \mathrm{~N}_{4}(\mathrm{SN})$ secondary particle network, $D_{q}(q=0,1,2)$, to investigate the relationship between the thermal conductivity and the connectivity of SN secondary particles in the SN/ SUS316L composites related to the propagation path of heat conduction, while we also highlighted the usefulness of the analysis of the multifractal dimensions of material textures in the solid-state reaction. ${ }^{14)}$ This report also outlined how the capacity dimension $D_{0}$ is related to the morphology of $\beta$ - $\mathrm{Si}_{3} \mathrm{~N}_{4}$ agglomerates, how the information dimension $D_{1}$ for these samples was related to the entropy of configuration affected by the dispersion of the $\beta-\mathrm{Si}_{3} \mathrm{~N}_{4}$ particles, and how the correlation dimension $D_{2}$ reflected the connectivity (or dispersibility) among the particles. ${ }^{14)}$

In this study, it is focused on the formation of the selfassembled BT secondary particle group in the BT/PVDF composites depending on the dispersant involved in the manufacturing process. Especially, the properties of the BT/PVDF composites were controlled by the self-assembly of the BT secondary particle groups. The multifractal dimensions and the dielectric properties of self-assembled BT/PVDF composites are investigated with the difference in the viscosity of the dispersant. The material texture of the self-assembled BT/PVDF composites by the selfassembly process and the $\mathrm{BT} / \mathrm{PVDF} / \mathrm{BT}$ heterointerface in the BT secondary particle groups were controlled by changing the viscosity of PEG. Multifractal analysis was adopted for the evaluation of the morphology and dispersion states of the self-assembled BT secondary particle groups under the different kneading conditions.

\section{Experimental procedures}

\subsection{Sample preparation}

The BT powder (Fuji Titanium Industry Co., Ltd., Japan, HPBT-1) used as the starting material had a particle diameter of approximately $0.58-0.75 \mu \mathrm{m}$ (D50). BT/PVDF composites were generated from the BT powder and PVDF powder (Arkema S.A., KYNAR ${ }^{\circledR} 711$ ), with the PVDF melted at a temperature of $483 \mathrm{~K}$ and the BT powder subsequently added to the PVDF to form a mixture. The volume fractions of the BT were $0,5,10$, and $20 \%$. The BT/ PVDF composites were mixed under $10 \mathrm{rpm}$ for $30 \mathrm{~min}$, with each sample cooled in air following mixing. To generate BT/PVDF composites with dispersion, both BT powder and polyethylene glycol (FUJIFILM Wako Pure Chemical Corporation, Japan, PEG [PEG1000, average molecular $\quad$ weight $=900-1,100 \mathrm{cst}, \quad$ viscosity $=15-35$ $\mathrm{mPa} \cdot \mathrm{s}$ at $\left.25^{\circ} \mathrm{C}\right]$ ) were mixed and kneaded under the same conditions as described above. The amount of additive PEG in the mixture was approximately $4 \mathrm{wt} \%$ of the BT 
powder. PEG was added to the BT/PVDF composite to disperse BT particles and aggregate them in the PVDF. To investigate the effect of the viscosity of the dispersion on the self-assembly process, the BT/PVDF composites were manufactured with PEG (1: PEG2000, FUJIFILM Wako Pure Chemical Corporation, Japan, average molecular weight $=1,800-2,200 \mathrm{cst}$, viscosity $=35-60 \mathrm{mPa} \cdot \mathrm{s}$ [at $25^{\circ} \mathrm{C}$ ], 2: PEG20000, Showa Chemical Co., Ltd., Japan, average molecular weight $=20,000,10,000-15,000$ cst [at $\left.99^{\circ} \mathrm{C}\right]$ ), respectively.

\subsection{Measurement method}

The $\varepsilon^{\prime}$ and the loss tangent $(\tan \delta)$ of the BT/PVDF composite at a frequency range of $100-110 \mathrm{MHz}$ were measured at $298 \mathrm{~K}$ using an Agilent 4294A precision impedance analyzer with lead wires made of platinum. Each sample presented a rectangle with a surface area of $70 \mathrm{~mm}^{2}$ and a thickness of $0.3 \mathrm{~mm}$. The electrodes were gold with square faces.

The cross-sections of the samples were analyzed using a scanning electron microscope (SEM, Hitachi High-Tech Co., Japan TM3000) and a field emission scanning electron microscope (FE-SEM, Hitachi Co., Japan: S-4100 and SU-8230, JEOL Ltd.: JSM-7001F). The SEM images were converted to binary images to estimate the $S$ of the BT particles for each sample. The average secondary particle area $(S)$ was estimated from the numbers of pixels obtained by dividing the total number of black pixels by the number of particle groups in the binary image.

\subsection{Multifractal analysis}

The morphology and dispersion of self-assembled BT/ PVDF composites were characterized using multifractal analysis, which was performed using the binary images. This analysis was used to estimate the probability $\left(p_{i}[r]\right)$ of an image by covering it with boxes of size $r$ and counting the number of boxes. The partition function is as follows:

$$
P_{r}^{q}=\sum_{i=1}^{N(r)}\left[p_{i}(r)\right]^{q}
$$

where $q$ is the order moment $(-\infty<q<\infty)$. The generalized dimensions of the $q$ of a distribution, $D_{q}$, can be defined as follows:

$$
D_{q}=\frac{1}{q-1} \lim _{r \rightarrow 0} \frac{\log P_{r}^{q}}{\ln r}=\frac{1}{q-1} \lim _{r \rightarrow 0} \frac{\log N(q, r)}{\ln r}
$$

When $q$ takes the values of 0,1 , or 2 , Eq. (2) is reduced to the following: ${ }^{15)}$

$$
\begin{aligned}
D_{0} & =-\lim _{r \rightarrow 0} \frac{\log N(r)}{\log r} \\
D_{1} & =\lim _{r \rightarrow 0} \frac{\sum_{i=1}^{N(r)} \mu_{i}(r) \log \left(\mu_{i}(r)\right)}{\log (r)} \\
D_{2} & =\lim _{r \rightarrow 0} \frac{\log (C(r))}{\log (r)}
\end{aligned}
$$

where $D_{0}$ is the capacity dimension, $C(r)$ is the correlation function, and $D_{1}$ and $D_{2}$ are the entropy dimension and the correlation dimension, respectively. The relationship between $D_{0}, D_{1}$, and $D_{2}$ is as follows:

$$
D_{2} \leq D_{1} \leq D_{0}
$$

In this paper, $D_{0}, D_{1}$, and $D_{2}$ were estimated to evaluate the morphology and dispersion of the self-assembled BT secondary particle groups in the BT/PVDF composite.

\section{Results}

Figure 1 presents the SEM micrograph and binary images of the BT/PVDF composites without the dispersant under low-speed kneading. In Fig. 1, the black part of the binary images shows the BT particle group, while the white part shows PVDF. As Figs. 1(a)-1(c) shows, the formation of self-assembled BT secondary particle groups
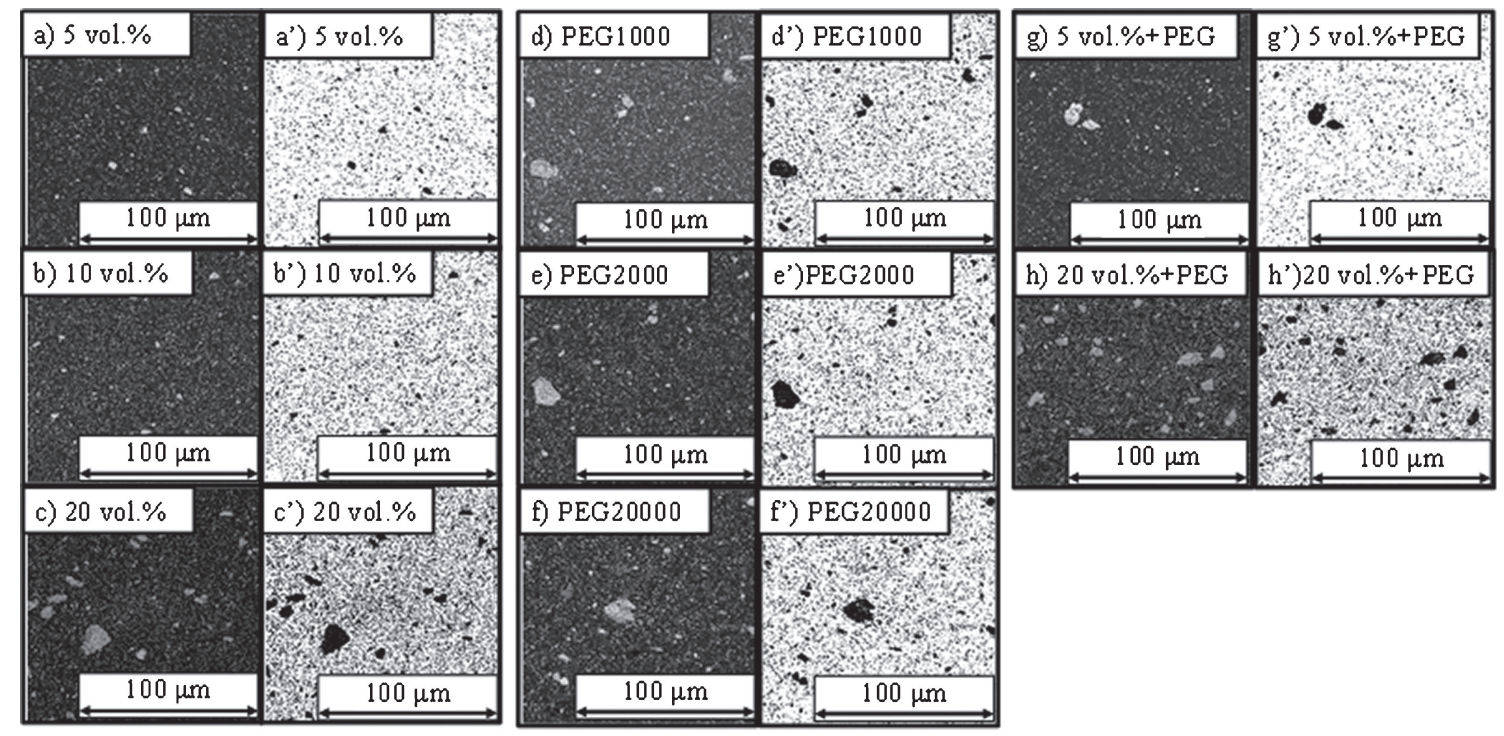

Fig. 1. Cross-sectional SEM images of BT/PVDF composites under low-speed kneading conditions with and without PEG1000, PEG2000, and PEG20000. 


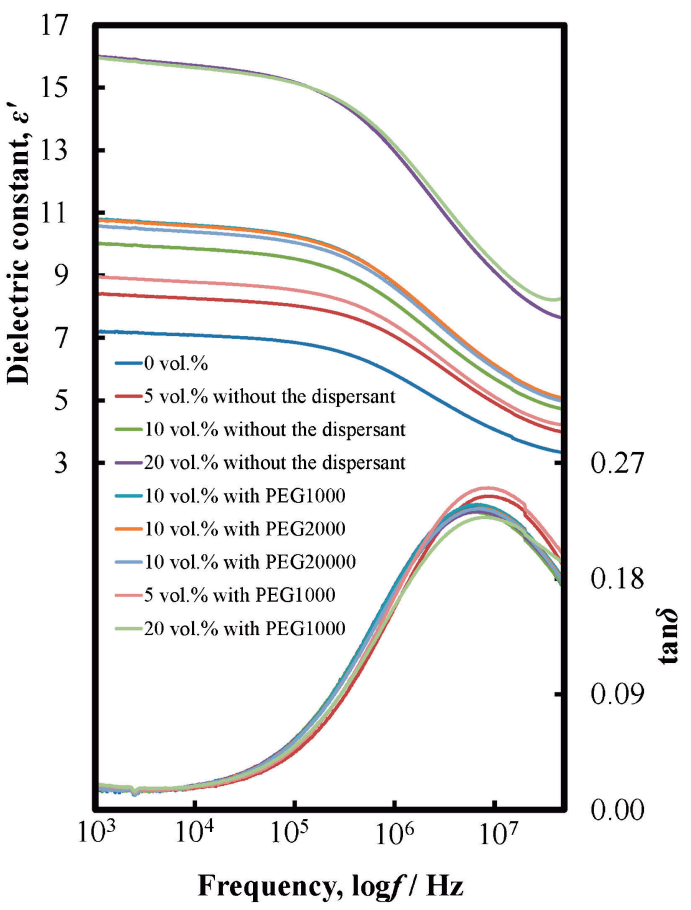

Fig. 2. Plots of $\varepsilon^{\prime}$ and $\tan \delta$ of BT/PVDF composites with and without PEG1000, PEG2000, and PEG20000 in relation to the frequency.

was promoted with the addition of BT particles, while as Figs. 1(d)-1(f) shows, the formation of self-assembled BT secondary particle groups was promoted with an increase in PEG viscosity. Meanwhile, as Figs. $1(\mathrm{~g})$ and $1(\mathrm{~h})$ show, the formation of self-assembled BT secondary particle groups of the BT/PVDF composites with PEG1000 was promoted with the addition of BT particles with a BT volume fraction of 5 and $20 \mathrm{vol} . \%$. However, the formation of self-assembled BT aggregates was not promoted from 10 to 20 vol. $\%$. Comparing the 10 vol. $\%$ BT particles, it was confirmed that self-assembled BT secondary particle groups were larger in the order of (1) the sample with additives of PEG20000, (2) the sample with additives of PEG2000, (3) the sample with additives of PEG1000, and (4) the sample without the dispersant. It was also found that the formation of self-assembled BT secondary particle groups was affected by viscosity due to the diffusion process of the self-assembly process.

Figure 2 shows the $\varepsilon^{\prime}$ in relation to the frequency dependence with the addition of BT powder. The $\varepsilon^{\prime}$ of the samples with and without PEG1000 with 20 vol.\% BT was the highest at all frequencies. Xia et al. ${ }^{16)}$ proposed the dielectric response of three PVDF phases of $\alpha, \beta$, and $\gamma$ as a function of frequency. Here, the authors reported that the dielectric loss $(\tan \delta)$ of $\alpha$-PVDF and $\gamma$-PVDF increased clearly at above $1 \mathrm{MHz}$ due to the dielectric relaxation. ${ }^{16)}$ As shown in Fig. 2, the maximum value of $\tan \delta$ was confirmed at $4 \mathrm{MHz}$, while it subsequently decreased at values above $4 \mathrm{MHz}$. Here, it is suggested that $\beta$-PVDF was partially formed by adding BT powder.

Figure 3 presents the results of the volume fraction of BT and the $\varepsilon^{\prime}$ at $1 \mathrm{kHz}$. To evaluate the effects of the filler,

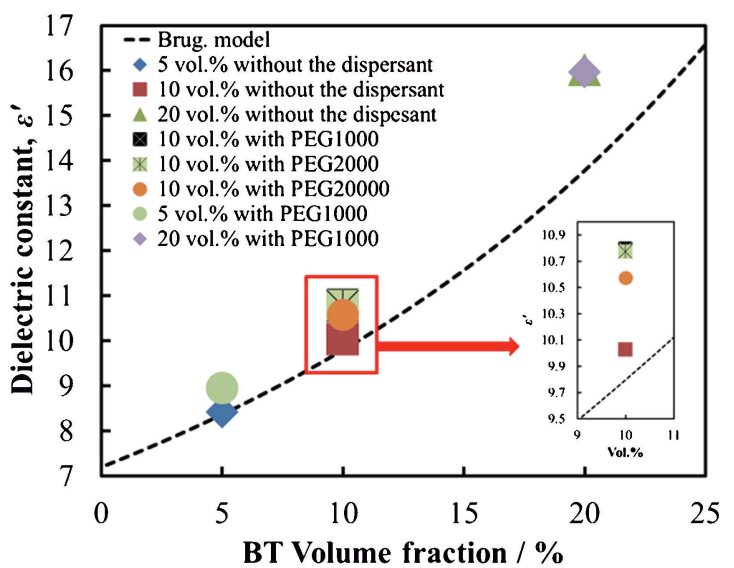

Fig. 3. $\varepsilon^{\prime}$ of $\mathrm{BT} / \mathrm{PVDF}$ composites at $1 \mathrm{kHz}$ in relation to the volume fraction of BT powders.

the Bruggeman model ${ }^{17)}$ was used with the composite materials, in which the fillers were homogeneously dispersed in the matrix, as shown in Fig. 3. The model can be described as follows: ${ }^{18)}$

$$
1-V=\frac{\varepsilon_{\text {composite }}^{\prime}-\varepsilon_{\text {filler }}^{\prime}}{\varepsilon_{\text {matrix }}^{\prime}-\varepsilon_{\text {filler }}^{\prime}}\left(\frac{\varepsilon_{\text {matrix }}^{\prime}}{\varepsilon_{\text {composite }}^{\prime}}\right)^{1 / 3}
$$

where $V$ is the volume fraction of $\mathrm{BT}, \varepsilon^{\prime}$ matrix is the $\varepsilon^{\prime}$ of $\operatorname{PVDF}\left(\varepsilon_{\text {matrix }}^{\prime}=7.2\right), \varepsilon_{\text {filler }}^{\prime}$ is the $\varepsilon^{\prime}$ of BT $\left(\varepsilon_{\text {filler }}^{\prime}=\right.$ $1,000)$, and $\varepsilon^{\prime}$ composite is the $\varepsilon^{\prime}$ of the BT/PVDF composite. The relationship between the viscosity of the dispersant and the $\varepsilon^{\prime}$ was then examined. In the 10 vol.\% BT particles, the $\varepsilon^{\prime}$ of the BT/PVDF without PEG was slightly higher than that of the Bruggeman model, while the $\varepsilon^{\prime}$ of the BT/PVDF composites with PEG was higher than that of the Bruggeman model. Comparing the $\varepsilon^{\prime}$ of the samples of $10 \mathrm{vol} . \%$ BT particles with and without PEG, it was confirmed that the $\varepsilon^{\prime}$ were in the order of (1) the sample with PEG1000, (2) the sample with PEG2000, (3) the sample with PEG20000, and (4) the sample without PEG. In this paper, samples of 5 and 20 vol.\% were prepared with PEG1000 since the $\varepsilon^{\prime}$ of the sample with PEG1000 was the highest among the samples with the dispersant. In the sample without PEG1000, the $\varepsilon^{\prime}$ of the 10 and 20 vol.\% samples was higher than that of the Bruggeman model. However, the $\varepsilon^{\prime}$ of the 5 vol.\% sample was the same as that of the Bruggeman model. This could have been due to the coarse (agglomeration) particle samples that can be explained in terms of the number of particles along the agglomerated low-impedance path. ${ }^{4}$ )

The morphology and dispersion of self-assembled BT secondary particle groups in the BT/PVDF composites were investigated using $D_{0}, D_{1}$, and $D_{2}$ estimated via multifractal analysis. Figure 4 shows the results for the generalization dimension of all samples, with $D_{0}, D_{1}$, and $D_{2}$ determined based on these results. Generally, the relationship between $D_{0}, D_{1}$, and $D_{2}$ is reported to be $D_{2} \leq D_{1} \leq D_{0} .{ }^{15)}$ However, the equality $D_{0}=D_{1}=D_{2}$ occurs only if the fractal is statistically or exactly selfsimilar and homogeneous. ${ }^{15}$ ) The data related to the $S$, the average $\varepsilon^{\prime}$, and $D_{0}, D_{1}$, and $D_{2}$ are shown in Table 1. In a 
Table 1. Characteristics of BT/PVDF composites

\begin{tabular}{|c|c|c|c|c|c|c|}
\hline $\begin{array}{l}\text { BT Volume fraction } \\
\qquad / \%\end{array}$ & & $\begin{array}{c}\text { Capacity } \\
\text { dimension }\left(D_{0}\right)\end{array}$ & $\begin{array}{c}\text { Entropy } \\
\text { dimension }\left(D_{1}\right)\end{array}$ & $\begin{array}{c}\text { Correlation } \\
\text { dimension }\left(D_{2}\right)\end{array}$ & $\begin{array}{c}\text { Dielectric } \\
\text { constant }\left(\varepsilon^{\prime}\right)\end{array}$ & $\begin{array}{c}\text { Average secondary particle area } \\
\text { of BT particles }(S) / \mu \mathrm{m}^{2}\end{array}$ \\
\hline \multirow[t]{2}{*}{5} & Without PEG1000 & $1.75(0)$ & $1.69(5)$ & $1.64(5)$ & 8.42 & 0.98 \\
\hline & With PEG1000 & $1.72(5)$ & $1.66(6)$ & $1.61(1)$ & 8.95 & 0.98 \\
\hline \multirow[t]{4}{*}{10} & Without PEG1000 & $1.85(7)$ & $1.81(1)$ & $1.77(1)$ & 10.03 & 1.10 \\
\hline & With PEG1000 & $1.84(3)$ & $1.78(9)$ & $1.74(4)$ & 10.80 & 1.29 \\
\hline & With PEG2000 & $1.84(2)$ & $1.79(0)$ & $1.74(6)$ & 10.77 & 1.36 \\
\hline & With PEG20000 & $1.83(4)$ & $1.78(2)$ & $1.73(9)$ & 10.57 & 1.47 \\
\hline \multirow[t]{2}{*}{20} & Without PEG1000 & $1.93(3)$ & $1.89(9)$ & $1.87(1)$ & 16.01 & 1.38 \\
\hline & With PEG1000 & $1.92(0)$ & $1.88(1)$ & $1.84(9)$ & 15.96 & 1.42 \\
\hline
\end{tabular}

The third decimal place in parenthesis contains an error.

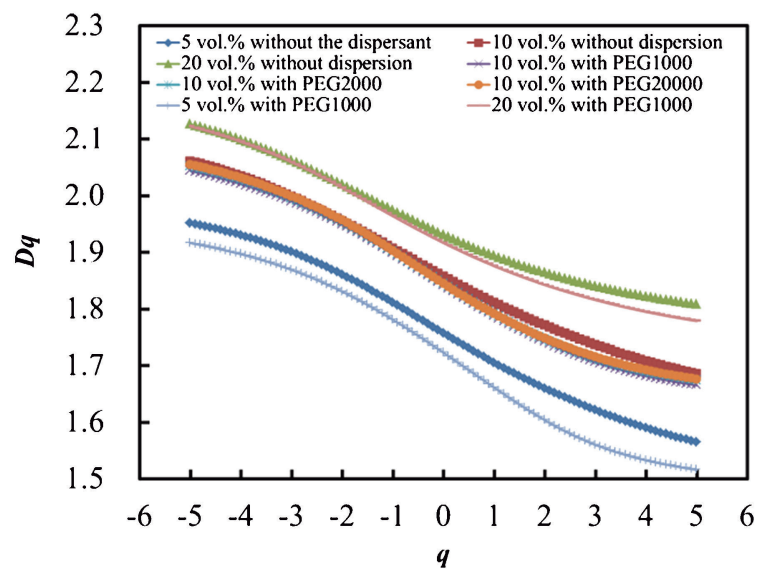

Fig. 4. Multifractal spectra curves $(D[q]-q)$ for BT/PVDF composites with and without PEG1000, PEG2000, and PEG20000.

previous report, $D_{0}$ was found to be related to the morphology of ceramic agglomerates, $D_{1}$ to the entropy of configuration affected by the dispersion of ceramic particles, and $\mathrm{D}_{2}$ to the dispersibility (or connectivity) among the particles. ${ }^{14)}$ The formation of the self-assembled BT secondary particle groups in relation to the viscosity of the dispersant was investigated by using multifractal analysis. The $D_{0}, D_{1}$, and $D_{2}$ of the samples with PEG1000 and PEG2000 were almost the same, while those of the sample with PEG20000 were very close to those of the PEG1000 and PEG2000 samples; however, the $D_{0}, D_{1}$, and $D_{2}$ of the sample with PEG20000 were slightly lower than those of the samples with PEG1000 and PEG2000. As such, it is suggested that the viscosity of the dispersant affected the formation of the secondary particle group of BT fillers.

\section{Discussion}

Figure 5 shows the results for the $S$ and $\varepsilon^{\prime}$. It has previously been reported that the $\varepsilon^{\prime}$ of self-assembled BT/ PLLA composites increases with an increase in the $S$ of BT particles when BT aggregates are formed via the selfassembly process in BT/PLLA composites. ${ }^{7)}$ Robertson and Varlow ${ }^{4)}$ suggested that a low-impedance path forms BT agglomerations, improving the $\varepsilon^{\prime}$. From the results shown in Fig. 5, it is clear that the $\varepsilon^{\prime}$ of the sample with and without PEG1000 increased with the formation of a self-assembled BT secondary particle group. Meanwhile,

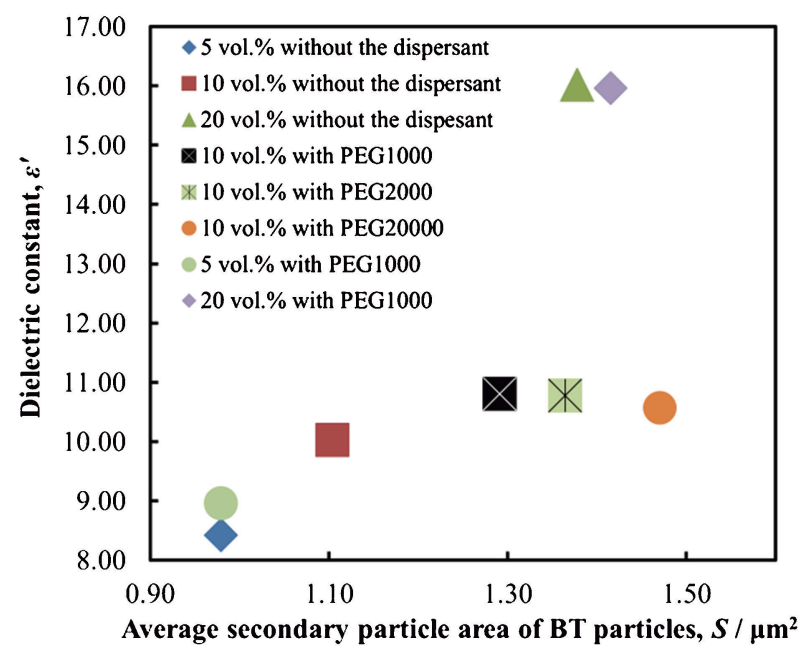

Fig. 5. Relationship between $\varepsilon^{\prime}$ and $S$ of BT particles.

comparing the $10 \mathrm{vol} \%$ BT samples, the $S$ of the BT particles of the samples increased with an increase in PEG viscosity.

The self-organization processes in a non-equilibrium chemical system have been studied in a solution system described by the Belousov-Zhabotinsky reaction. ${ }^{19)}$ The self-organization process is observed in the reactiondiffusion system, which is assumed to occur due to a competitive reaction between an inhibitor and a promoter within the diffusion process. The reaction can be described by the following equation:

$$
\partial v / \partial t=u-v+D_{v} \nabla^{2} v
$$

where $u$ is the active term, $v$ is the inhibitive term, and $D_{v}$ is the diffusion coefficient. Equation (9) presents the selfassembly process of a solid based on Eq. (8): ${ }^{8)}$

$$
\text { Aggregation } \Leftrightarrow \text { Decomposition }+ \text { Diffusion process }
$$

This reaction corresponds to a process of aggregation and decomposition. It was suggested that the cohesive force in this process was the intermolecular force. After mixing the BT powder and the dispersant to cover the surface of the BT powder, the BT powder was added to the PVDF. The dispersant was dissolved in the PVDF. Furthermore, the BT and PVDF were bonded, and it was considered that intermolecular force worked as a cohesion 
force to reduce the surface energy of BT particles to form BT aggregates. The shear stress induced by the stirring speed acts in terms of decomposition. The kneading process can indicate the diffusion process in the reactiondiffusion system. Generally, when particles are mixed in a viscous liquid, the diffusivity of the particles is affected by the viscosity of the liquid. The viscosity of the dispersant was changed as the kneading condition in this paper. As such, it is suggested that the aggregation was advanced in the self-assembly process with the increase in PEG viscosity, while the formation of self-assembled BT secondary particle groups was promoted.

Comparing the 10 vol.\% BT samples with PEG1000, PEG2000, and PEG20000, the $\varepsilon^{\prime}$ s of the samples with PEG1000 and PEG2000 were close to equal. However, the $\varepsilon^{\prime}$ was decreased by increasing PEG viscosity, which means the $\varepsilon^{\prime}$ was affected by the viscosity of the dispersant while the $S$ of the samples was increased by increasing the viscosity of PEG. On comparing the volume fractions of BT powder in the samples with and without PEG1000, the $S$ of BT particles of the 10 and $20 \mathrm{vol} . \%$ BT samples were found to be the same, while the number of self-assembled BT secondary particle groups was increased by adding BT powder.

To investigate the formation of self-assembled BT aggregates with the $\mathrm{BT} / \mathrm{PVDF} / \mathrm{BT}$ heterointerface resulting from the change in the viscosity of the dispersant, FE-SEM was performed for samples with and without PEG1000, PEG2000, and PEG20000 with 10 vol.\% BT, with the resulting images shown in Fig. 6. Figures 6(a) and 6(a') show the sample without PEG, while Figs. 6(b) and 6(b'), Figs. 6(c) and 6(c'), and Figs. 6(d) and 6(d') present the samples with PEG1000, PEG2000, and PEG20000, respectively. The BT powder is indicated by the blue arrow and the PVDF by the red arrow. In the sample without PEG [Figs. 6(a) and 6(a')], it was confirmed that the selfassembled BT aggregates had a BT/PVDF/BT heterointerface in the BT secondary particle groups. However, the formation of voids between the BT and PVDF was confirmed. In the sample with PEG1000 [Figs. 6(b) and 6(b')], it was confirmed that the BT particles and the PVDF were aggregated in the secondary particle groups. Furthermore, the self-assembled BT aggregates with a BT/PVDF/BT heterointerface were predominantly formed in the secondary particle groups. The air might enter the BT/PVDF composites in the kneading process in air. In a previous report, ${ }^{8)}$ the dielectric properties of the self-assembled BT/PLLA composites were investigated. The dielectric constant of air is lower than PLLA and BT. However, the dielectric constant of the BT/PLLA composites having the BT/PLLA/BT heterointerface was higher than the Bruggeman model. Furthermore, the $\tan \delta$ of the BT/PLLA composite having the BT large granules was high because the BT particles were not bonded. The $\tan \delta$ of the BT/ PLLA composite having the BT/PLLA/BT heterointerface in the BT aggregates was lower than that of the BT/PLLA composite having the $\mathrm{BT}$ large granules. It was considered that the voids at the BT/PLLA interface of the BT aggre-

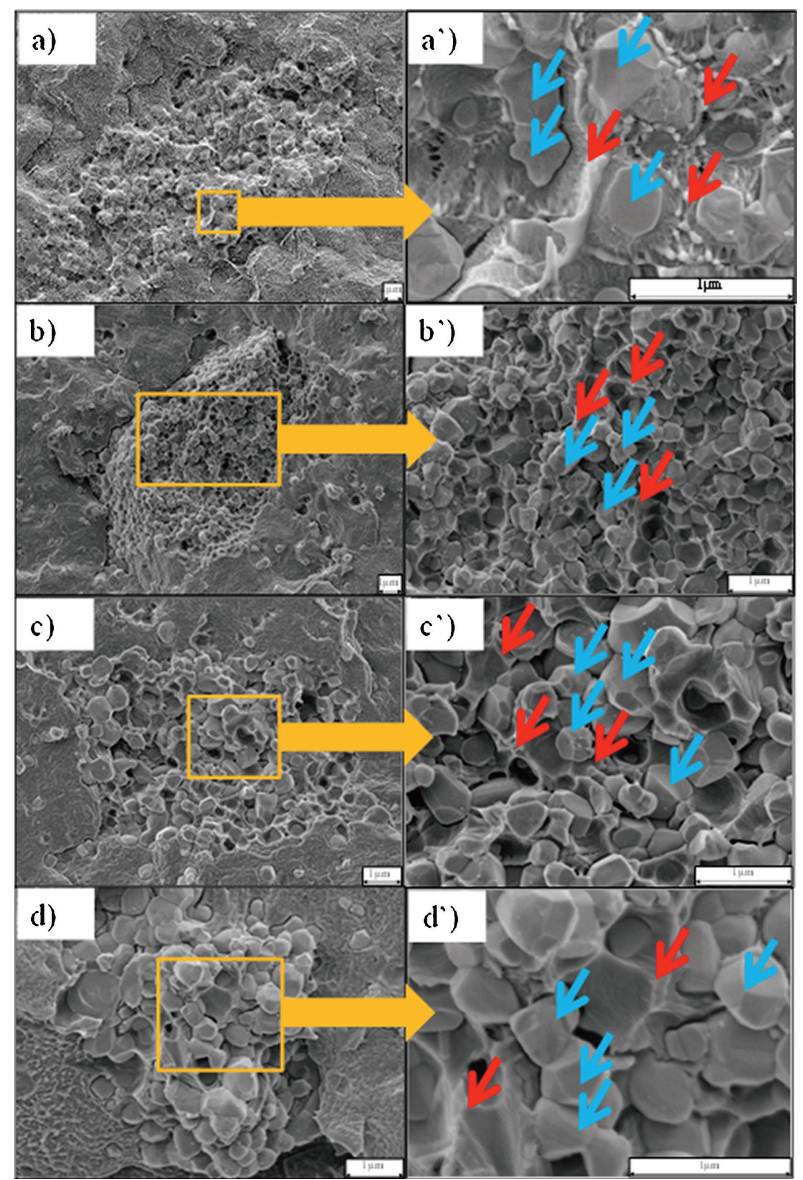

Fig. 6. Cross-sectional FE-SEM images and magnified images of BT/PVDF with and without PEG1000, PEG2000, and PEG20000, with 10 vol.\% fraction of BT.

gates did not efficiently reflect the dielectric properties. Therefore, it was suggested that the dielectric properties of the BT/PVDF composites were not affected efficiently by the void in this paper. In the sample with PEG2000 [Figs. 6(c) and 6(c')], the formation of self-assembled BT aggregates with a BT/PVDF/BT heterointerface was confirmed. However, here, a BT/BT interface was also formed. Finally, in the sample with PEG20000 [Figs. 6(d) and $\left.6\left(\mathrm{~d}^{\prime}\right)\right]$, the formation of self-assembled BT aggregates with a BT/PVDF/BT heterointerface was confirmed. However, here, the BT/BT interface was dominantly formed. In this paper, the BT particles were covered with PEG in advance, and the BT powder was added to the PVDF. In the case of adding highly viscous PEG to the BT/PVDF composite, the resistance to decompose the $\mathrm{BT}$ particles was increased. The PEG then dissolved into the PVDF, and the BT agglomerates with the $\mathrm{BT} / \mathrm{BT}$ interface were formed in the BT/PVDF composite. On the other hand, in the viscosity of PEG is low, the resistance to decompose was low. It was suggested that the BT particles were decomposed and aggregated. Hence, the formation of the BT/PVDF/BT heterointerface was promoted. Therefore, it is suggested that the PEG viscosity affected the formation of the BT/ PVDF/BT heterointerface. As such, the sample with PEG1000 had BT aggregates, the sample with PEG20000 


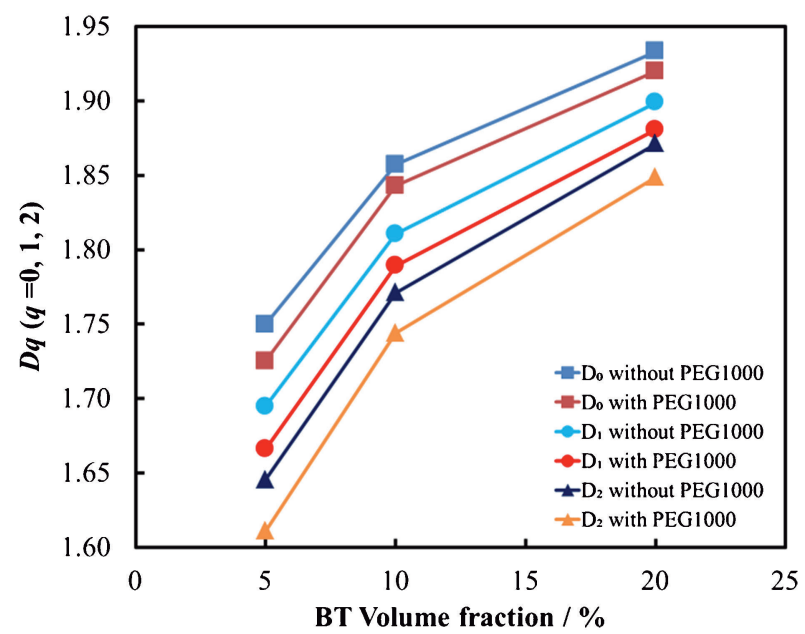

Fig. 7. $D_{q}(q=0,1$, and 2$)$ of BT/PVDF composites in relation to the volume fraction of BT powders.

had BT agglomerates, and the sample with PEG2000 had a mixture of BT aggregates and agglomerates. In a previous report, it was suggested that the electric dipole of a BT/ polymer/BT heterointerface can be induced by improving the $\varepsilon^{\prime}{ }^{8)}$ In fact, when BT granules with a BT/BT interface were added, the $S$ was found to be large, while the change in the $\varepsilon^{\prime}$ was found to be saturated. ${ }^{8}$ In our results for the $S$ and $\varepsilon^{\prime}$ of the samples with PEG2000 and PEG20000 (Fig. 5), it was clear that the $S$ increased with the increase in $\mathrm{PEG}$ viscosity. However, the $\varepsilon^{\prime}$ decreased, which was likely due to the formation of the BT/BT interface (Fig. 6). As such, it is suggested that the viscosity of the dispersant affected the formation of the BT/PVDF/BT heterointerface and that the heterointerface plays an important role in improving the $\varepsilon^{\prime}$. As Table 1 shows, the self-assembled BT agglomerates with PEG20000 had a predominant BT/BT interface, while the $D_{1}$ and $D_{2}$ of the sample were lower than those of the PEG1000 and PEG2000 samples. It is thus suggested that the viscosity of the dispersant affected the self-assembly process and that the multifractal dimension was affected by the viscosity.

To investigate the effect of the BT fillers, the $D_{0}, D_{1}$, and $D_{2}$ of the samples with and without PEG1000 were examined, with the results shown in Fig. 7. Here, the $D_{0}$ of the samples increased with the addition of BT powder. Therefore, it is suggested that the morphology of the selfassembled BT secondary particle groups with and without PEG1000 depended on the volume fraction of the BT powder. On comparing the samples with and without PEG1000, it was confirmed that the $D_{0}$ of the samples without PEG1000 was higher than that of the sample with PEG1000. This was likely because the morphology of the self-assembled BT secondary particle groups with the dispersant was more anisotropic than the samples without the dispersant. As Fig. 7 shows, the $D_{1}$ of the samples with and without PEG1000 was increased by adding the BT powders. On comparing the samples with and without PEG1000, it was confirmed that the $D_{1}$ of the samples with PEG1000 was lower than that of the samples without
PEG1000, with the configuration entropy likely decreased with the addition of the PEG1000. That is, the formation of BT secondary particle groups was promoted by adding PEG1000. Meanwhile, the $D_{2}$ was estimated to investigate the dispersibility of the BT secondary particle groups. The $D_{2}$ of the samples with and without PEG1000 was increased by increasing the volume fraction of the BT particles. On comparing the sample with and without PEG1000, it was confirmed that the $D_{2}$ of the samples with PEG1000 was lower than that of the samples without PEG1000. As such, it is suggested that the bias was reduced. As Fig. 7 shows, the $D_{1}$ and $D_{2}$ decreased with the addition of PEG1000. This indicated that the distribution was biased by adding the PEG1000 and that the formation of self-assembled BT secondary particle groups was promoted. The $D_{0}, D_{1}$, and $D_{2}$ of the samples with PEG1000 approached those of the samples without PEG1000 with the addition of BT powder. Here, it is suggested that the morphology and distribution of BT secondary particle groups with and without PEG1000 were approached with the addition of the BT powder. Therefore, the $\varepsilon^{\prime}$ and the $S$ of the self-assembled BT secondary particle groups of the samples with and without PEG1000 of 20 vol.\% BT were almost the same.

\section{Conclusions}

To control the formation of self-assembled BT secondary particle groups, the dispersants of the difference viscosity were added. The samples without the dispersant, samples with PEG1000, samples with PEG2000, and samples with PEG20000 of 5-20 vol.\% BT were prepared. The conclusions are as follows:

(1) The $\varepsilon^{\prime}$ of the BT/PVDF composites with selfassembled BT aggregates was higher than that of the Bruggeman model. The $\varepsilon^{\prime}$ increased with an increase in the $S$ of the BT particles. The $S$ of the BT particles increased in accordance with the increase in PEG viscosity. However, the change in $\varepsilon^{\prime}$ was saturated.

(2) On comparing the $S$ of the BT particles with PEG1000, PEG2000, and PEG20000, the formation of self-assembled BT secondary particle groups was found to have been promoted with the increase in PEG viscosity. Here, it can be concluded that the viscosity of the dispersant affected the self-assembly process.

(3) On comparing the results of the multifractal analysis for the samples with PEG1000, PEG2000, and PEG20000, the $D_{0}, D_{1}$, and $D_{2}$ of the sample with PEG20000 were found to be slightly lower than those of the samples with PEG1000 and PEG2000. Here, it can be concluded that the viscosity of the dispersant affected the self-assembly process and the multifractal dimension was affected by the viscosity.

(4) The FE-SEM results for the samples with PEG1000, PEG2000, and PEG20000 indicated that the formation of a $\mathrm{BT} / \mathrm{PVDF} / \mathrm{BT}$ heterointerface was promoted with the addition of the lowest PEG viscosity. That is, the formation of self-assembled BT aggregates was dependent on the viscosity of the dispersant. 
(5) On comparing the samples with and without PEG1000, the morphology and distribution of selfassembled BT secondary particle groups were found to be different. That is, it was indicated that the distribution was biased by adding PEG1000, while the formation of selfassembled BT secondary particle groups was promoted.

Hence, BT aggregates were formed in the sample with PEG1000, BT agglomerates were formed in the sample with PEG20000, and both aggregates and agglomerates were formed in the sample with PEG2000. The viscosity of the dispersant affected the self-assembly process, with the multifractal dimension subsequently reflecting this.

It was suggested that the multifractal properties and dielectric properties of the BT/PVDF composites were possible to be controlled by self-assembly of BT aggregates. It was found that the formation of the BT aggregates was contributed to improving the dielectric properties of the BT/PVDF composites. In particular, the BT/PVDF/ $\mathrm{BT}$ heterointerface in the BT aggregates played an important role in improving the dielectric properties of the BT/ PVDF composites.

Acknowledgment I am grateful to Prof. Yue Bao (Tokyo City University) and Ms. Yuka Mizukami (Tokyo City University) for their assistance with the multifractal analysis. The work was supported by the "Joint Research Center for Environmentally Conscious Technologies in Materials and Science" at the Joint Usage/Research Center at Kagami Memorial Research Institute for Materials Science and Technology at WASEDA UNIVERSITY. This work was partly supported by TOKYO CITY UNIVERSITY Interdisciplinary Research Center for Nano Science and Technology for instrumental analysis.

\section{References}

1) P. Barber, S. Balasubramanian, Y. Anguchamy, S. Gong, A. Wibowo, H. Gao, H. J. Ploehn and H. C. Zur Loye, Materials, 2, 1697-1733 (2009).
2) N. Sakamoto, H. Yoshioka, J. Suzuki and T. Suzuki, J. Ceram. Soc. Jpn., 118, 669-673 (2010).

3) M. Takeda, K. Yahagi, T. Hirata, T. Kuroiwa, C. Nakajima, Y. Suzuki and F. Munakata, IEEJ T. Sensor. Micromachines, 140, 43-49 (2020).

4) J. Robertson and B. R. Varlow, IEEE T. Dielect. El. In., 12, 779-790 (2005).

5) Y. Akimune, F. Munakata, M. Ando, Y. Okamoto and N. Hirosaki, J. Ceram. Soc. Jpn., 105, 122-125 (1997).

6) T. T. M. Phan, N. C. Chu, V. B. Luu, H. Nguyen Xuan, I. Martin and P. Carriere, Compos. Part A-Appl. S., 90, 528-535 (2016).

7) F. Munakata, K. Yoshino, K. Nemoto, S. Abe and A. Ito, Mater. Lett., 221, 147-149 (2018).

8) M. Takeda, K. Yoshino, A. Ito, M. Tanimura, Y. Inoue, Y. Koyama and F. Munakata, J. Ceram. Soc. Jpn., 129, 135-142 (2021).

9) S. Mihcitaka, M. Yohikane, H. Mitsuaki and O. Toshino, J. Soc. Powder Technol., 25, 11-15 (1988).

10) S. Kobayashi, R. Kobayashi and T. Watanabe, Acta Mater., 102, 397-405 (2016).

11) M. Takeda, K. Yoshino, Y. Mizukami, Y. Sato, A. Ito, Y. Bao and F. Munakata, J. Jpn. Soc. Powder Powder Metall., 66, 122-127 (2019).

12) R. Lopes and N. Betrouni, Med. Image Anal., 13, 634649 (2009).

13) E. Pérez, C. Bernal and M. Piacquadio, Appl. Surf. Sci., 258, 8940-8945 (2012).

14) F. Munakata, M. Takeda, K. Nemoto, K. Ookubo, Y. Sato, Y. Mizukami, M. Koga, S. Abe, Y. Bao and R. Kobayashi, J. Alloy. Compd., 853, 156570 (2021).

15) A. Posadas, R. Quiroz, A. Tannús, S. Crestana and C. M. Vaz, Nonlinear Proc. Geoph., 16, 159-168 (2009).

16) W. Xia and Z. Zhang, IET Nanodielectrics, 1, 17-31 (2018).

17) A. Lakhtakia, B. Michel and W. S. Weiglhofer, Compos. Sci. Technol., 57, 185-196 (1997).

18) H. Looyenga, Physica, 31, 401-406 (1965).

19) I. R. Epstein, J. A. Pojman and O. Steinbock, Chaos, 16, 037101 (2006). 